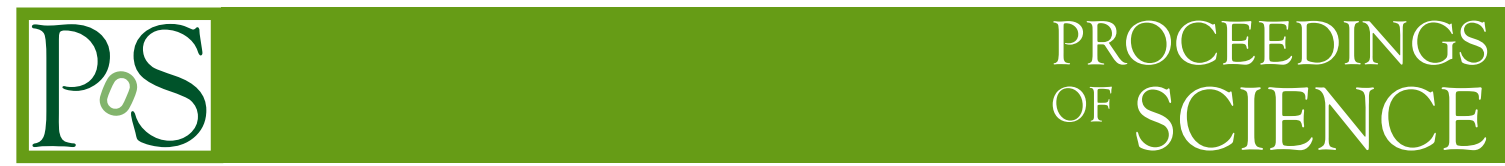

\title{
The astrophysics of LIGO gravitational-wave observations
}

\section{Ilya Mandel*}

Birmingham Institute for Gravitational Wave Astronomy and School of Physics and Astronomy, University of Birmingham, Birmingham, B15 2TT, United Kingdom

E-mail: imandelestar.sr.bham.ac.uk

We very briefly summarize the possible formation channels of merging binary black holes detected by LIGO and comment on future prospects in gravitational-wave astrophysics.

11th INTEGRAL Conference Gamma-Ray Astrophysics in Multi-Wavelength Perspective, 10-14 October 2016

Amsterdam, The Netherlands

${ }^{*}$ Speaker. 
At the time of the INTEGRAL2016 meeting, where the talk summarised here was originally presented, the LIGO Scientific Collaboration and Virgo Collaboration had announced two confident and one further probable gravitational-wave detections $[1,2,3]$. Since then, a further discovery has been announced as of July 2017 [4]. All discoveries to date correspond to mergers of pairs of black holes, with total masses between $\sim 20$ and $\sim 60$ solar masses. This proves that binary black holes exist; that they merge; and that they are rather heavy, extending to higher masses than any of the known black holes in X-ray binaries with confident dynamical mass measurements [5, 6].

The high masses of the black holes indicate that the stars that formed them were born in lowmetallicity environments, where stellar winds are suppressed, allowing massive stars to retain more mass. This could either indicate formation in local low-metallicity pockets, or in the early Universe with very long delay times between star formation and black hole merger [7].

A black hole merger within the age of the Universe through energy loss by gravitational-wave emission points to an initial separation was less than about a fifth of an astronomical unit [8]. This is much smaller than the typical size to which progenitor stars are expected to expand during the giant phase. This raises a few possibilities for the formation scenario:

- Binaries start in wide orbits in the field, go through one or more mass transfer phases as the stars expand, and are brought closer (where gravitational-wave emission can be efficient) when orbital energy is used to eject the common envelope. This classical isolated binary evolutionary channel has been explored over several decades; a few of the many relevant references include [9], [10], and [11].

- Massive stars that start in close binaries in low-metallicity environments are kept rapidly spinning through tidal locking, allowing for efficient mixing and chemically homogeneous evolution. Such stars fuse nearly all of their hydrogen into helium, do not expand, and form black holes in situ, close enough for gravitational wave emission to be efficient [12, 13].

- The two black holes could form independently before being brought together by a series of dynamical encounters in a dense stellar environment. Dynamical formation in either globular clusters $[14,15]$ or galactic nuclear clusters $[16,17]$ could contribute to the population of LIGO detections.

- More exotic proposed channels include the formation and merger of two black holes during the collapse of a core of a single very massive star [18] or mergers of primordial black holes arising from early-Universe cosmological perturbations [19].

The population of gravitational-wave observations of binary mergers, which will grow rapidly as LIGO detectors become more sensitive, will make it possible to address the inverse problem of gravitational-wave astrophysics: how to go from a population of observed sources to understanding key uncertainties about binary evolution?

One possible approach is exploring the large parameter space of astrophysical models to determine which are consistent with the observed population [20,21]. Typically, the model predictions are too expensive to evaluate over the full range of parameters, so this will require the development of new tools to emulate model predictions [6]. An alternative, weakly-modeled approach is to carry out clustering on the detected sources in order to search for subpopulations [22, 23]. 
By demanding consistent models to explain compact object mergers, supernovae, gamma ray bursts, Galactic double neutron stars, X-ray binaries including Be X-ray binaries, luminous red novae, etc., we can move toward a concordance picture of the evolution of massive stars in binaries.

\section{References}

[1] B. P. Abbott, R. Abbott, T. D. Abbott, M. R. Abernathy, F. Acernese, K. Ackley et al., Observation of Gravitational Waves from a Binary Black Hole Merger, Physical Review Letters 116 (Feb., 2016) 061102, [1602.03837].

[2] B. P. Abbott, R. Abbott, T. D. Abbott, M. R. Abernathy, F. Acernese, K. Ackley et al., GW151226: Observation of Gravitational Waves from a 22-Solar-Mass Binary Black Hole Coalescence, Physical Review Letters 116 (June, 2016) 241103, [1606.04855].

[3] B. P. Abbott, R. Abbott, T. D. Abbott, M. R. Abernathy, F. Acernese, K. Ackley et al., Binary Black Hole Mergers in the First Advanced LIGO Observing Run, Physical Review X 6 (Oct., 2016) 041015, [1606.04856].

[4] B. P. Abbott, R. Abbott, T. D. Abbott, F. Acernese, K. Ackley, C. Adams et al., GW170104: Observation of a 50-Solar-Mass Binary Black Hole Coalescence at Redshift 0.2, ArXiv e-prints (June, 2017), [1706.01812].

[5] F. Özel, D. Psaltis, R. Narayan and J. E. McClintock, The Black Hole Mass Distribution in the Galaxy, Astrophysical Journal 725 (Dec., 2010) 1918-1927, [1006.2834].

[6] W. M. Farr, N. Sravan, A. Cantrell, L. Kreidberg, C. D. Bailyn, I. Mandel et al., The Mass Distribution of Stellar-mass Black Holes, Astrophysical Journal 741 (Nov., 2011) 103, [1011. 1459 ].

[7] M. Dominik, E. Berti, R. O'Shaughnessy, I. Mandel, K. Belczynski, C. Fryer et al., Double Compact Objects III: Gravitational-wave Detection Rates, Astrophysical Journal 806 (June, 2015) 263, [1405.7016].

[8] B. P. Abbott, R. Abbott, T. D. Abbott, M. R. Abernathy, F. Acernese, K. Ackley et al., Astrophysical Implications of the Binary Black Hole Merger GW150914, Astrophysical Journal 818 (Feb., 2016) L22, [1602.03846].

[9] A. V. Tutukov and L. R. Yungelson, The merger rate of neutron star and black hole binaries, MNRAS 260 (Feb., 1993) 675-678.

[10] V. M. Lipunov, K. A. Postnov and M. E. Prokhorov, Formation and coalescence of relativistic binary stars: the effect of kick velocity, MNRAS 288 (June, 1997) 245-259, [astro-ph / 9702060 ].

[11] K. Belczynski, D. E. Holz, T. Bulik and R. O'Shaughnessy, The first gravitational-wave source from the isolated evolution of two stars in the 40-100 solar mass range, Nature 534 (June, 2016) 512-515, [1602.04531].

[12] I. Mandel and S. E. de Mink, Merging binary black holes formed through chemically homogeneous evolution in short-period stellar binaries, MNRAS 458 (May, 2016) 2634-2647, [1601.00007].

[13] P. Marchant, N. Langer, P. Podsiadlowski, T. M. Tauris and T. J. Moriya, A new route towards merging massive black holes, A\&A $\mathbf{5 8 8}$ (Apr., 2016) A50, [1601.03718].

[14] S. Sigurdsson and L. Hernquist, Primordial black holes in globular clusters, Nature 364 (July, 1993) 423-425. 
[15] C. L. Rodriguez, C.-J. Haster, S. Chatterjee, V. Kalogera and F. A. Rasio, Dynamical Formation of the GW150914 Binary Black Hole, Astrophysical Journal 824 (June, 2016) L8, [1604. 04254 ].

[16] I. Bartos, B. Kocsis, Z. Haiman and S. Márka, Rapid and Bright Stellar-mass Binary Black Hole Mergers in Active Galactic Nuclei, ArXiv e-prints (Feb., 2016), [1602.03831].

[17] N. C. Stone, B. D. Metzger and Z. Haiman, Assisted inspirals of stellar mass black holes embedded in AGN discs: solving the 'final au problem', MNRAS 464 (Jan., 2017) 946-954, [1602 .04226].

[18] A. Loeb, Electromagnetic Counterparts to Black Hole Mergers Detected by LIGO, Astrophysical Journal 819 (Mar., 2016) L21, [1602 .04735].

[19] S. Bird, I. Cholis, J. B. Muñoz, Y. Ali-Haïmoud, M. Kamionkowski, E. D. Kovetz et al., Did LIGO Detect Dark Matter?, Physical Review Letters 116 (May, 2016) 201301, [1603 . 00464 ].

[20] S. Stevenson, A. Vigna-Gómez, I. Mandel, J. W. Barrett, C. J. Neijssel, D. Perkins et al., Formation of the first three gravitational-wave observations through isolated binary evolution, Nature Communications 8 (Apr., 2017) 14906, [1704.01352].

[21] S. Stevenson, C. P. L. Berry and I. Mandel, Hierarchical analysis of gravitational-wave measurements of binary black hole spin-orbit misalignments, ArXiv e-prints (Mar., 2017) , [1703.06873].

[22] I. Mandel, C.-J. Haster, M. Dominik and K. Belczynski, Distinguishing types of compact-object binaries using the gravitational-wave signatures of their mergers, MNRAS 450 (June, 2015) L85-L89, [1503.03172].

[23] I. Mandel, W. M. Farr, A. Colonna, S. Stevenson, P. Tiňo and J. Veitch, Model-independent inference on compact-binary observations, MNRAS 465 (Mar., 2017) 3254-3260, [1608 . 08223]. 